\section{Planning Estratégico desde la Semiótica y el Pragmatismo de Pierce}

Jairo Roberto Sojo Gómez *

Resumen: La ponencia a presentar aborda algunos conceptos teóricos del pragmatismo y la semiótica de Charles Sanders Peirce, en relación con el signo, las relaciones que se establecen entre signos y su proyección a la Planeación Estratégica Publicitaria - Planning. A partir de dichos elementos teóricos se propone un modelo semiótico de planeación estratégica, resultado del proyecto de investigación: "Semiótica, planeación y estrategia publicitaria. Proyecciones del Pragmatismo a la comunicación contemporánea" financiado por la Universidad Jorge Tadeo Lozano y ejecutado en 2014-2015. La pregunta que orienta la ponencia es: ¿Cuáles elementos del Pragmatismo y la semiótica peirceana pueden proyectarse a un modelo de planeación estratégica en publicidad? Para el desarrollo de la ponencia se trabajan tres argumentos fundamentales del pragmatismo, conocidos como categorías fenomenológicas, las cuales se relacionan con las sensaciones, las acciones y los pensamientos que se suscitan en la mente humana. Se relacionan entre el sentir de las personas, la acciones que hacen y el pensamiento de esas acciones y que en el ejercicio publicitario se asocian con las sensaciones que tienen los consumidores frente a los productos o marcas, las acciones de compra y los procesos de filiación con las marcas (branding estratégico). Este planteamiento secuencial permite proponer una metodología de planeación estratégica publicitaria atada a resultados medibles y eficientes. El modelo de Planeación Estratégica Publicitaria - Planning, se usó con gran éxito en 4 casos EFFIE College 2016 en Colombia. El resultado en esta competencia dio: 2 finalistas y un Oro en el caso del cliente Bancolombia.

Palabras clave: Semiótica - Peirce - publicidad - modelo de planeación estratégica.

[Resúmenes en inglés y portugués en las páginas 166 - 167]

(*) Magister en Publicidad, Especialista en Psicología del Consumidor y Profesional en Publicidad de La Tadeo. Coordinador del Programa de Publicidad en Cartagena de Indias en la Universidad Jorge Tadeo Lozano. Dicta en pregrado asignaturas de planeación estratégica y creatividad y varios seminarios de posgrado en la especialización de gerencia de publicidad. Tiene más de 15 años de experiencia en el área de cuentas y planeación estratégica en agencias como DDB Colombia, J. Walter Thompson, Indexcol, ADE y HAVAS. Ganador como tutor de un Effie College de oro y dos finalistas en el 2017, nominado como Profesor de Publicidad del año 2016 por la Revista P\&M y nombrado entre Los Líderes de la Publicidad y el Mercadeo para el 2020 por la misma revista. 


\section{Introducción}

Atrás quedó tratar a las personas como targets o clicks. Hoy se buscan nuevas formas de hacer Planning y se encontró una valiosa partiendo del pensamiento filosófico de Charles Sanders Pierce. Se plantea un modelo de relaciones tríadicas que funciona encontrando sensaciones, acciones y pensamientos de personas desde la semiótica.

La presente ponencia hablará acerca del pragmatismo partiendo de la tesis del profesor Vladimir Sánchez, "La construcción del sentido publicitario: Modelo de análisis y evaluación desde la perspectiva semeiótica de Charles Sanders Peirce", donde se plantea un modelo de relaciones tríadicas que funcionan para la planeación estratégica en cuanto a las comunicaciones publicitarias. Para ello se comienza con el siguiente planteamiento:

¿Cuáles elementos del Pragmatismo y la semiótica peirceana pueden proyectarse a un modelo de planeación estratégica en publicidad?

Este es el punto de inicio. En los siguientes apartes se partirá del pragmatismo para profundizar un poco más en el tema y adentrarnos en la base del nuevo modelo de planeación publicitaria.

"Todo anuncio publicitario tiene como objetivo final generar y movilizar representaciones, remisiones e interpretaciones deseables y favorables frente a bienes, productos, servicios, personas e ideologías. El proceso anteriormente descrito, es posible de ser analizado e interpretado a la luz de las teorías semióticas y en particular desde las propuestas de Charles Sanders Peirce. En efecto, sobre la base teórica de Peirce es posible comprender el proceso de la relación entre el signo, el objeto al que representa y el interpretante que media, que subyace a la manifestación de sentido en la pieza publicitaria. Dicho proceso es entendido como un acto de producción de sentido, que se da gracias a la acción, o influencia, que es, o implica, una cooperación de tres sujetos, a saber, un signo, su objeto y su interpretante". (Sánchez, V. septiembre 2011).

Al tener este análisis semiótico y al ponerlo al servicio publicitario enlazando estos dos mundos se puede predecir que abrirá muchas puertas. La inclusión de la planeación estratégica a las empresas ligadas a la publicidad está llena de incertidumbres. La industria publicitaria tiene en su mira tomar las tendencias y la innovación y transformarla en planeación sin tener los soportes indicados. Por eso es muy peligroso llamar planning a cualquier investigación, novedad o tendencia que aparece a la luz en el mercado o en las redes. Hoy se ve en las agencias colombianas que la planeación estratégica se convierte en uno de los pilares más importantes de ingresos propios y los anunciantes empezaron a incorporar en las InHouse planners sacándolos de las agencias. Este momento tan decisivo en la industria publicitaria abre puertas a nuevas opciones en modelos de planeación que presenten formatos totalmente diferentes, sólidos y con soportes, motivando nuevos puntos de vista. Los clientes valorarán este nuevo modelo de planning ya que está soportado desde un novedoso proceso basado en la semiótica que presenta nuevos horizontes. 
El modelo fue puesto a prueba en los EFFIE College 2016 capítulo Colombia. Uno de ellos es el caso del cliente Bancolombia, que luego se convirtió en Effie de Oro. Se planteó desde el punto de vista de los millenials colombianos donde perciben con desconfianza la utilización de los servicios que prestan las entidades financieras. El desafío de la marca a las Universidades que participaron fue: ¿cómo lograr que los jóvenes abran su cuenta de ahorro $y$ realicen transacciones con las tarjetas de Bancolombia? Este fue el reto de comunicación de Bancolombia, el cual fue asumido por el equipo de trabajo que bajo este modelo de planeación estratégica presentó la campaña "Más que vivo". Luego de determinar los procesos del modelo que se explicarán a continuación, se presentó una campaña que consiste en un sorteo donde los millenials que abran su cuenta de ahorros o realicen transacciones periódicas con Bancolombia podrán cumplir el sueño de viajar y tener experiencias extremas alrededor del mundo. A cambio de ello, el ganador entregará al banco las fotos, audios y vídeos de la experiencia, con el fin que este material se convierta en las piezas publicitarias de Bancolombia. Así el cliente reducirá así el gasto de producción publicitaria, al tiempo que la posiciona la marca como confiable ya que apoya los sueños de sus clientes, siendo esta una estrategia gana-gana entre el banco y el cliente financiero. Un caso del modelo de planeación exitoso.

\section{Desarrollo de la ponencia}

\section{Las relaciones tríadicas (categorías fenomenológicas) en el planteamiento es- tratégico.}

En la primera parte de este desarrollo de la ponencia se tomarán apartes del estudio del pragmatismo de la tesis del profesor Vladimir Sánchez con base al pensamiento filosófico de Charles Sanders Pierce, donde se plantea un modelo de relaciones tríadicas que funciona para la planeación estratégica en cuanto a publicidad:

Una persona pragmática es alguien que está dispuesta a resolver asuntos de forma inmediata, que no se complica y más bien atiende lo que resulta útil y vital en ese momento, independientemente de sus consecuencias y sus antecedentes. El pragmatismo se le asocia al hecho de no tener principios y más bien atender a los medios.

Partiendo de la línea Pierciana, surgen tres argumentos fundamentales de los postulados del pragmatismo, conocidas como las categorías fenomenológicas las cuales tienen que ver con la relación entre el sentir del individuo, la acción que hacen los individuos, y el pensamiento de esas acciones:

1. La idea del razonamiento que está ligado a la idea de generalidad o PRIMERIDAD.

2. La importancia del significado y el significado vinculado al objeto o SEGUNDIDAD.

3. La relación entre creencia y acción o TERCERIDAD.

En breves palabras la idea primera que se tiene es: “¿Cómo se razona?”, la respuesta a ello es que se razona por secuencia. La segunda idea es “QQué se razona? $\mathrm{O}$ ¿Qué se piensa cuando se razona?", pues se piensan cosas del mundo, en las que entran los objetos, las personas y los eventos. Y la idea final es “¿Cuál es el objeto del pensamiento?”, el resultado implica a la idea de la representación. 
Dicho lo anterior, este proceso de razonamiento ligado a la generalidad se ubica en el modelo de planeación publicitaria con el término de Primeridad, "La primeridad es el modo de ser de aquello que es como es, positivamente y sin referencia a ninguna otra cosa". Es decir, es el reconocimiento de una cualidad. Entendiendo cualidad como una posibilidad de un hecho real (Reconocer).

"Una publicidad en primeridad es la publicidad que le interesa movilizar o cuyas respuestas del target son respuestas que tienen que ver con la sensación. Osea lo que al cliente lo que le interesa, más que vender productos por el momento o cualquier otra cosa es que la gente sepa que él existe, que la gente sepa que ese producto existe, y que tenga una primera sensación con ese producto". (Sánchez, V. 18 agosto de 2015).

En la segunda categoría fenomenología, la importancia del significado y el significado vinculado al objeto, se debe determinar principalmente “¿Qué es el significado?”. Las personas desde pequeñas han tenido una formación en el significado, desde el colegio era más enfocado a lo tradicional, a la lengua y la lingüística; donde se sitúa la primera parte de lo que se considera como el significado, que no es más ni menos que la definición de una palabra, su contenido (en pocas palabras, cuando se encuentra el significado de las cosas por medio del diccionario). Esta consideración hace que se piense que un objeto es algo fijo, estable, ya acordado y definitivo; en el que no hay la necesidad de cambiarle nada; como si fuera un significado único de todo.

En el modelo, el efecto que se quiere dar con la estrategia es que el consumidor conozca el objeto que en este caso sería el producto. A partir de ello el significado y el objeto se ubican en el modelo de planeación publicitaria con el término de Segundidad, "es el modo de ser de aquello que es como es, con respecto a una segunda cosa pero con independencia de toda tercera". Es decir, es una acción (entendiendo la acción como un esfuerzo realizado por el receptor o consumidor). Ej. Activaciones de marca en donde se tiene contacto directo con el objeto (promover).

"En la categoría de la segundidad puede haber casos en los cuales los clientes conocen sus productos, la gente tiene unas sensaciones frente al producto pero, la gente no compra el producto, entonces la acción publicitaria tiene que estar en caminada necesariamente a lo que podríamos decir de forma muy genérica al -call to action- de que haya una respuesta de reacción efectiva y real, es decir, de que la gente no solamente tome la decisión de compra sino que además pues quizá compre el producto”. (Sánchez, V. 18 agosto de 2015).

En la tercera categoría fenomenológica, La relación entre creencia y acción, se debe de unificar la concepción de significado con creencia, pues son igualmente conceptos que permiten conocer objetos, solo que en otro modo de hablar. La concepción que se tiene de creencia, logra repercusiones prácticas en la existencia. Lo que define una creencia es que tiene un efecto sobre el comportamiento y sobre la acción. 
En el modelo la creencia y la acción se ubican en el modelo de planeación publicitaria con el término de Terceridad, "La terceridad es el modo de ser de aquello que es tal como es, al relacionar una segunda y tercera cosa entre sí". Es decir, el reforzamiento de lo logrado anteriormente con la primeridad y segundidad; esto se hace por medio de cualidades ya adoptadas por una cultura, es decir por medio de convenciones (fidelizar).

"En tercer momento la publicidad puede darse en el caso en el que el cliente sabe a ciencia cierta cuales son las cualidades que tiene ese producto, es posible incluso que tenga un buen flujo de caja pero quizás el producto no tiene una afectividad de marca adecuada seguramente cuando se piensa en la marca, entonces por lo tanto la acción publicitaria que hay que desarrollar con ese cliente no es la acción para que la gente conozca el producto, sino la acción para que la gente se fidelice con el producto y tenga una afectividad con esa marca”. (Sánchez, V. 18 agosto de 2015).

\section{Modelo de planeación estratégica de relaciones triádicas.}

2.1. Objetivo de campaña: Para iniciar el proceso en el modelo de planeación, se define objetivo de comunicación como:

"El objetivo establece la meta o finalidad de la publicidad a realizar. Identifica el resultado final del proceso y define el papel específico de la publicidad en la resolución del problema planteado por el cliente. Por pequeño o grande que sea el presupuesto, sean cuales sean los medios de difusión, el objetivo responde a la cuestión básica y escencial: qué tiene que conseguir el anuncio". (Kavounas A. 2013)

En el proceso de ejecución del modelo de planeación, el cliente llega con un objetivo claro. Este expresa la necesidad del cliente o su deseo relacionado con la marca o el producto. En esta parte del proceso es importante validar este objetivo acorde a la investigación o experiencia que el equipo de la cuenta haya tenido anteriormente. Este objetivo se valida teniendo en cuenta el contexto mercado.

Se debe analizar el objetivo que tenga el cliente, si cumple con las necesidades reales y da solución al problema que plantea el cliente o si dicho objetivo se debe adaptar, ya que según el propósito planteado se llevará a cabo el inicio de la investigación semiótica que alimentará el modelo de planeación. Si no se plantea el objetivo de manera cuidadosa desde el comienzo, puede existir la posibilidad que los resultados que se arrojen al final no sean los esperados ni solucionen el problema inicial del cliente.

2.2. Diagnóstico de mercado: Se debe interpretar el sentido de los datos recopilados en la investigación que ha traído el cliente o que se han realizado para la preparación de la planeación estratégica. No se debe quedar con los datos tal como aparecen, se debe dar valor a detalles que se ven o no se ven. Se realizan estudios cualitativos, cuantitativos, se revisan estadísticas, visitas de campo, brand reviews, focus groups, cibergrafías, escuchas 
digitales, etc con el fin de aportar o cruzar datos y determinar nueva información más allá de la aparente.

Los tres pilares básicos son:

- Segmentación: Definir el destinatario al que se le pretende llegar con la marca, al que se le esta planeando la publicidad. Los aspectos más importantes a tener en cuenta en este diagnóstico de las personas son sus actitudes, opiniones y comportamientos, tanto en general como hacia la categoría.

- Categoría de mercado: El objetivo es observar a la competencia directa y a la que "funciona como sustituto" supliendo la necesidad que el producto o servicio cumple. Tanto la competencia directa, la indirecta como la sustituta son fundamentales para el análisis. Es importante también determinar en donde y en qué canales está la marca en relación a las demás. - Estrategias de comunicación: En este aspecto final del diagnóstico se observa la competencia: que cualidades resalta, cuales ventajas competitivas comunica, en que tono y estilo le habla al target. La herramienta fundamental en este proceso es el copy análisis. En este momento se realizan mapas de posicionamiento que serán de gran ayuda para la planeación.

"Los mapas de percepción, que escriben Luis Castro y Jesus Caldeiro son un instrumento de visualización y análisis de posicionamiento de una marca en términos de imagen, beneficios o situaciones de uso (entre otras variables) en el contexto competitivo en el que participa. El mapa se dibuja, se traza una cruz y así tenemos dos ejes de coordenadas que delimitan 4 campos, de modo que situamos en ellos unos puntos que indican dónde se creen que se situan las marcas o dónde se quiere colocar la propia” (Moliné M. 2000)

2.3 Proceso Semiótico: Para el caso de este modelo de planeación se entiende como el resultado del diagnóstico que se plantea como un problema o una oportunidad de la marca, en este punto del proceso empieza a determinar la relación entre lo que la marca significa para la persona, los objetos o hechos que la representan y lo que la persona interpreta o comprende de la representación. Con este análisis se construye una posible significación.

- Cadenas de Significación: Se entiende como la cadena del significante donde el sentido en que las personas expresan las ideas, hacen que le den un valor importante a su experiencia o conocimiento o relación con la marca. Se debe tener presente que los elementos de la cadena tienen una significación propia. El deslizamiento de la cadena de significación da pistas de lo que se implanta como realidad en las personas. En el momento del proceso de significación debe ser cuidado de los desvíos, ya que de un primer desvío nace un proceso de otras cadenas de significantes.

- Enciclopedia: Conjunto de saber de términos y definiciones que se relacionan con la categoría de la marca. Estos son necesarios para entender el imaginario del grupo de personas $\mathrm{y}$ del contexto en que las viven y entienden. 
- Verbatims: Es una metodología de investigación en la que se recogen frases de las entrevistas con un grupo de personas y tienen un peso importante para la planeación. Sirve para entender a las personas en su entorno natural y en relación con las demás personas. Esta información se obtiene mediante entrevistas, encuestas o escuchas digitales en redes sociales bajo la técnica de minería de datos.

\subsection{Ground: Idea Clave}

"Es el fundamento del signo, que es algo que está por algo para alguien, es decir cumple una función de representar otra cosa, remitir a una idea o generar un significado, pero siempre lo hace de algún aspecto, no se puede hacer de todo, hay una cualidad que es la que interesa representar, es el fundamento, el ground, una buena pieza publicitaria requiere la identificación clara del ground (esto está en el ámbito de la planeación), pero también que se logre representar adecuadamente este ground en un signo (esto está en el ámbito de lo creativo)". En un signo pueden identificarse 2 objetos diferentes, el objeto dinámico que es el objeto real que existe, por ejemplo, una cerveza, pero también el objeto inmediato, que es la representación de la cualidad que logramos identificar que puede ser la cualidad más importante del objeto, es decir es la manera como se representa la cualidad en las personas, y esa cualidad que queremos representar es el ground del signo. (Sánchez, V. 2017).

La función del signo, cuando pasa a ser ground, es expresar en una frase la esencia de lo que satisface la marca. Debe estar redactada de forma que inspire al Departamento Creativo. En muchos casos (casi todos) el ground queda fuera de la vista de las personas, ya que su función es trasmitir el pensamiento del análisis semiótico que garantiza que el estímulo atrae y compromete tocando las fibras sensibles que generarán una acción ya sea en primeridad, segundidad o terceridad como se verá en el siguiente punto.

2.5 Estrategia de análisis de ubicación: En esta fase empieza la estrategia. Al analizar los resultados de los diagnósticos y estudios semióticos previos encontramos que ya se tiene el Ground o idea clave. Es el momento que enuncia Moliné: "El estratega no vive a la espera de los estímulos, sino anticipándolos y creándolos sin parar”. (Moliné, 2000, p. 465), es en lo que se empieza a trabajar, a anticipar y a crear.

Para determinar en que momento está la marca o producto se debe analizar el Ground y así asignarle un momento para poder determinar las acciones a tomar que mostrarán los cuadrantes. El ground dará la entrada a la planeación en el modelo. Se analiza si el ground dicta: qué se quiere comunicar, si lo que se quiere comunicar va en relación de una cualidad (PRIMERIDAD), una acción (SEGUNDIDAD) o con el fin de fidelizar (TERCERIDAD). Así se ubica horizontalmente en el modelo de planeación: 
- Primeridad - Reconocer: Esta primera etapa responde al hecho de que el grupo de personas conozca la existencia del producto. Si este es el caso, se debe situar en primeridad para solucionar el problema. También aplica al caso de querer relacionar una cualidad especifica que ha dictado el ground con el producto o marca.

- Segundidad - Promover: En este punto de partida, las personas ya conocen la marca, incluso tiene percepciones al respecto. Puede ser que no lo encuentran o no lo compran. El ground, gracias al análisis semiótico dará una respuesta efectiva y real para que en la asociación las personas no solo den cuenta de la existencia del producto, sino que lo asocien a un signo. Generalmente por la experiencia con el modelo y con clientes, si existen problemas relacionados con la compra, se debe situar el producto en segundidad para que el modelo determine una acción a cumplir.

- Terceridad - Fidelizar: Finalmente se encuentran situaciones en las que las personas pueden que conozca el producto y sus atributos, este rote bien por los canales de distribución y sin embargo no existe un sentimiento de afecto por la marca. El ground puede encontrar signos que construyan una fidelización de las personas hacia la marca. Una recomendación del modelo es que se empiece a construir un hábito, y para esto lo situamos en terceridad.

\subsection{Modelo de Relaciones Tríadicas}

- La primera (Signo que es) tiene en cuenta el signo en si mismo.

- La segunda (Relación) tiene en cuenta la relación del signo con su objeto.

- La tercera (Como lo representa el interpretante) tiene en cuenta como el interpretante lo representa como posibilidad, hecho o razón.

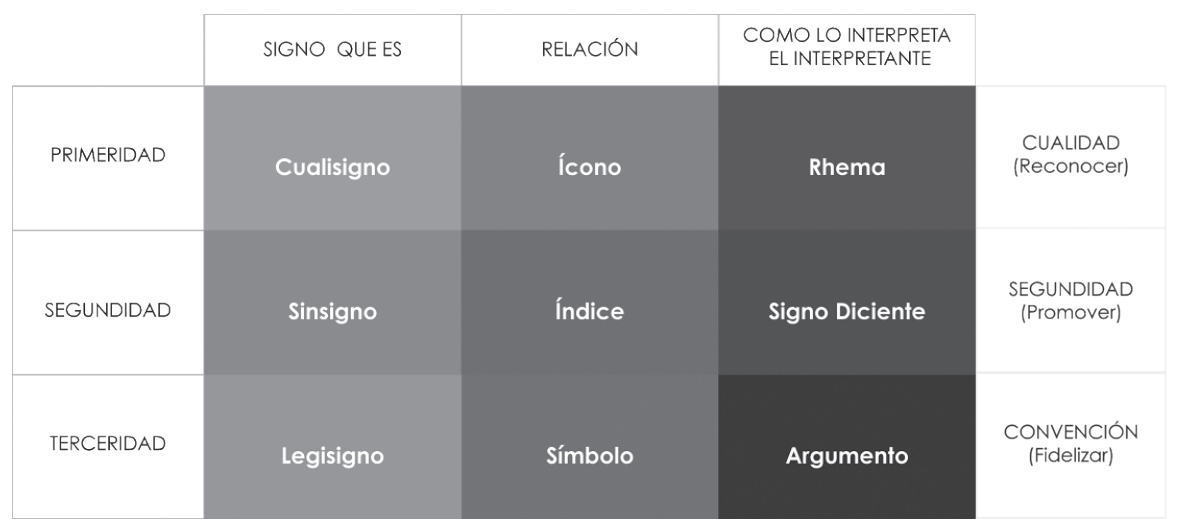

Según Peirce, estas relaciones se pueden dividir en tres tricotomías: 


\begin{tabular}{|c|c|}
\hline $\begin{array}{l}\text { PRIMERIDAD } \\
\text { "La primeriad es el modo de } \\
\text { ser de aquello que es como es, } \\
\text { positivamente y sin referencia } \\
\text { a ninguna otra cosa." }\end{array}$ & $\begin{array}{c}\text { CUALIDAD } \\
\text { La primeriad es el reconocimiento de una } \\
\text { cualidad (entendiendo cualidad como una } \\
\text { posibilidad de un hecho real) } \\
\text { CUALIDAD (Reconocer) }\end{array}$ \\
\hline $\begin{array}{l}\text { SEGUNDIDAD } \\
\text { "La segundidad es el modo de } \\
\text { ser de aquello que es como es, } \\
\text { con respecto a una segunda } \\
\text { cosa pero con independencia } \\
\text { de toda tercera." }\end{array}$ & $\begin{array}{c}\text { ACCIÓN } \\
\text { La segundidad es una acción (entendiendo la } \\
\text { acción como un esfuerzo realizado por el receptor) } \\
\text { Ej. Activaciones de marca en donde se tiene } \\
\text { contacto directo con el objeto. } \\
\text { ACCIÓN (Promover) }\end{array}$ \\
\hline $\begin{array}{l}\text { TERCERIDAD } \\
\text { "La segundidad es el modo de } \\
\text { ser de aquello que es como es, } \\
\text { con respecto a una segunda } \\
\text { cosa pero con independencia } \\
\text { de toda tercera." }\end{array}$ & $\begin{array}{c}\text { CONVENCIÓN } \\
\text { La terceridad es el reforzamiento de lo logrado } \\
\text { anteriormente con la primeridad y segundidad; esto se } \\
\text { hace por medio de cualidades ya adoptadas por una } \\
\text { cultura, es decir por medio de convenciones. } \\
\text { CONVENCIÓN ( Fidelizar) }\end{array}$ \\
\hline
\end{tabular}

Estas tricotomías del eje horizontal se cruzan con las columnas verticales de acción:

\begin{tabular}{|c|c|c|}
\hline $\begin{array}{c}\dot{\mathrm{Q} Q U E ́ ~ S E} \\
\text { QUIERE COMUNICAR? }\end{array}$ & 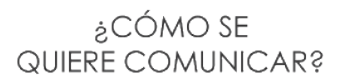 & RESPUESTA ESPERADA \\
\hline
\end{tabular}

Para efectos de la publicidad el modelo de relaciones triádicas, toma sentido en comunicación en cada una de las casillas, así:
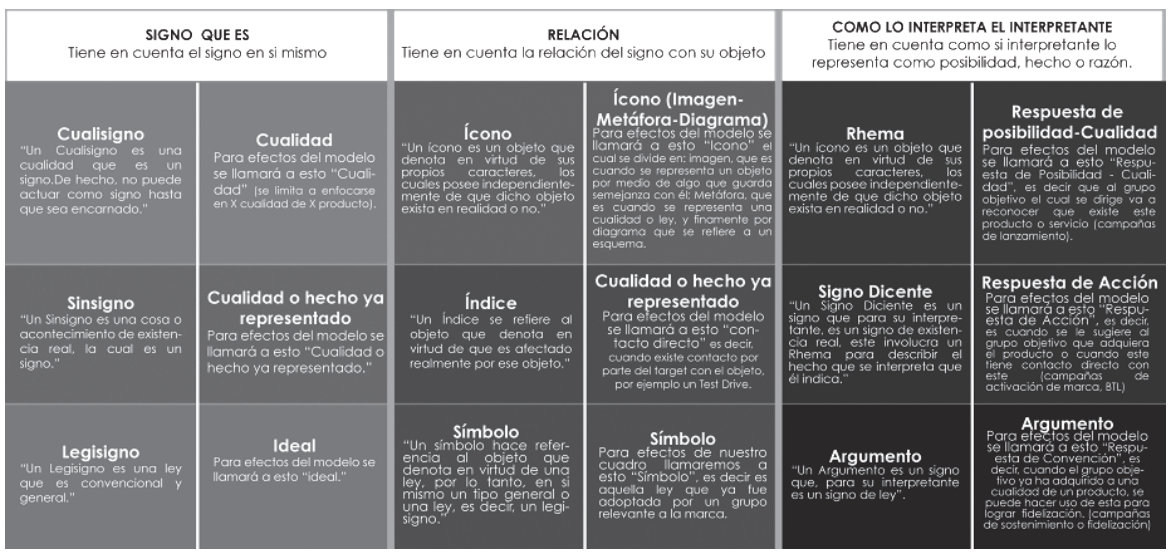
En el lenguaje publicitario, la primera columna la llamaremos ¿Qué quiere comunicar? La segunda ¿Cómo se quiere comunicar? y finalmente la tercera columna es la - Respuesta esperada - por parte del interpretante.

Teniendo estas reflexiones claras, se analiza el proceso con la entrada del ground en el modelo de planeación: (Posibilildades)

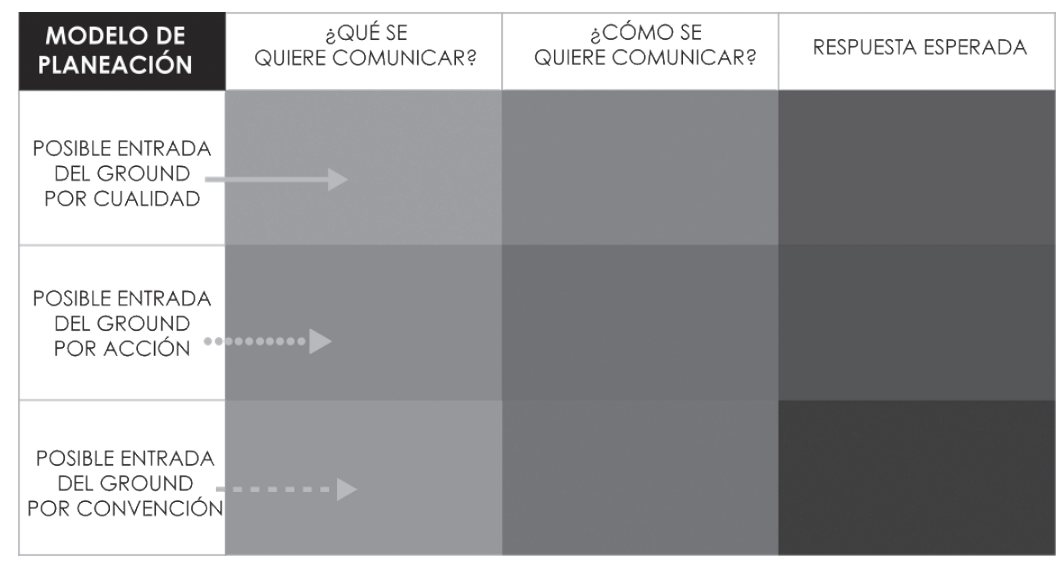

- Ya con el ground definido y su entrada al modelo, se empieza a analizar el camino en Cualidad (o primeridad):

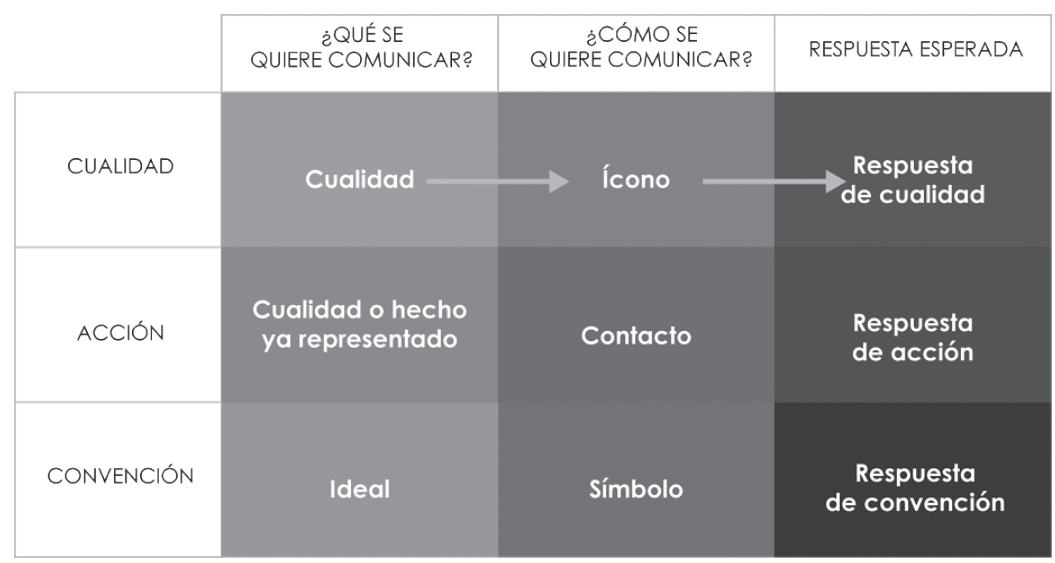

Este camino que se vislumbra en planeación, indica que la marca necesita trabajar sobre una cualidad definida en el proceso semiótico que es representada por un ícono y que las personas responderán emocionalmente a esa cualidad comunicada. 
- Con el ground definido, existen las siguientes posibles combinaciones de acción (o segundidad):

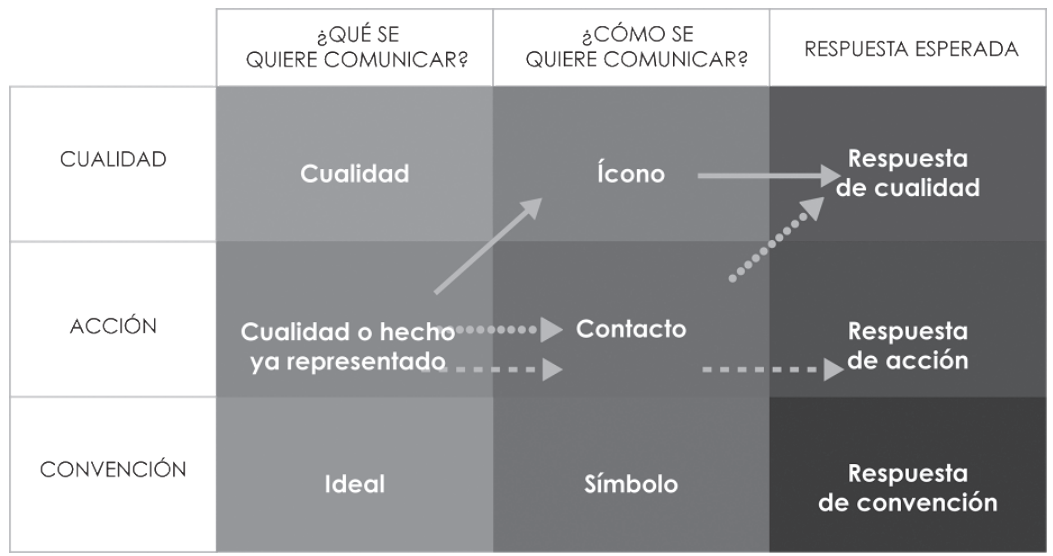

Con el ground apuntando en segundidad - acción , se presentan 3 opciones. Se debe trabajar en la propuesta de planeación en determinar una cualidad o hecho ya representado, que quiere decir que se tiene que lograr un primer contacto, acercamiento o abordaje a las personas en una activación de marca por ejemplo. Una de las opciones dependiendo del ground, puede tener un ícono para la comunicación o proponer ideas para generar un contacto "uno a uno, un BTL" con las personas. La respuesta se tendrá en las personas que valoran la cualidad publicitada o en acciones claras "call to actions" de las personas respondiendo a la planeación.

- Para un ground en terceridad, estas son las posibles combinaciones de Convención (o terceridad):

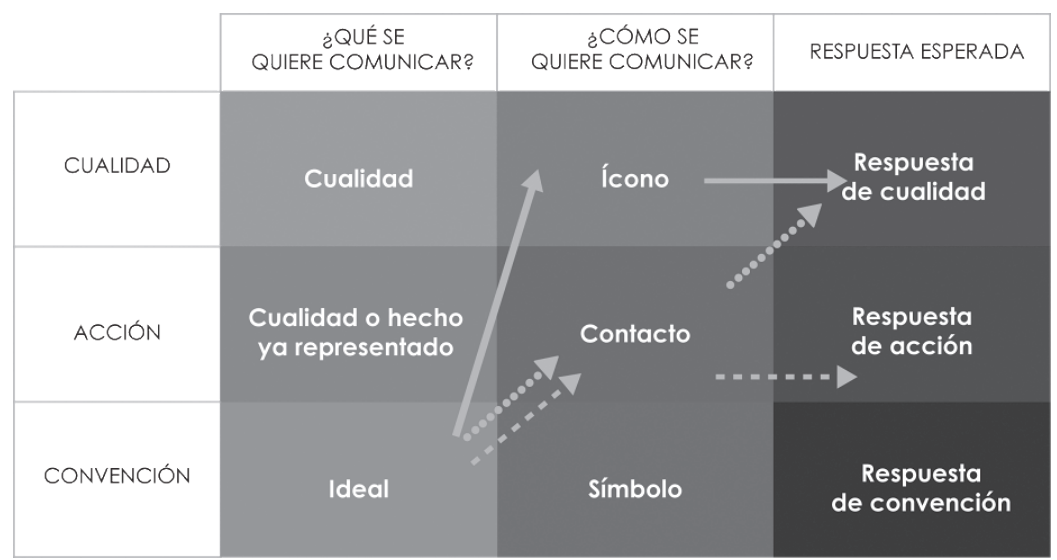


Otras posibles combinaciones de Convención (o terceridad) desde "Ideal":

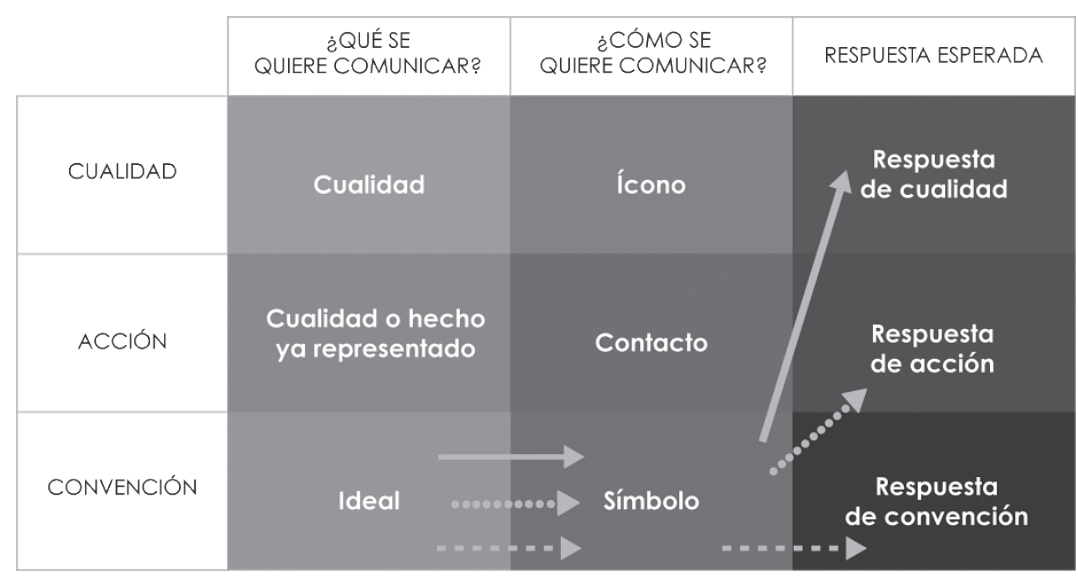

En este caso, cuando el ground marca Convención tiene varias opciones. Se analizará el camino que apunta el Ideal, ya que han sido explicado los anteriores caminos. En Ideal indica que se debe tener una comunicación convencional que será direccionada con un símbolo que representa la marca. De este punto de la planeación puede terminar en las 3 referencias de respuesta siendo la respuesta de convención, acciones de fidelización de las personas hacia la marca.

La planeación estratégica se marca según los movimientos generados por Pierce. Es un proceso que ayuda a visualizar y diseñar el plan a futuro sobre lo que va a lograr el ground en la marca. Involucra direccionamientos sobre qué decir, qué elementos usar en la comunicación y cuál es la respuesta que vamos a recibir según la entrada del ground en el modelo de planeación. También asegura el cumplimiento del objetivo de marca, para aprovechar al máximo el potencial existente de comunicación y sobre todo aprovechar el proceso semiótico que hace diferente este modelo de planeación.

Es muy importante tener una visión específica del modelo de planeación en el momento que según el movimiento, dicta la ruta exacta para tener un rumbo fijo y así darle sentido a los objetivos. Con esto en la mira, se establecen condicionantes, lineamientos y procesos al departamento creativo que llevarán a cumplir los objetivos en el plazo determinado y con una eficiencia grarantizada. Siempre se tendrá la posibilidad de mejorar y afinar el modelo durante las diversas fases.

\section{Conclusión}

¿No sería increíble si pudiera predecir el futuro con un alto grado de precisión? Todo se haría diferente. Más en la publicidad que experimenta cambios minuto a minuto y hace que se nublen los objetivos a mediano y corto plazo. Desde las nuevas visiones de Burnett, 
Reeves, Ogilvy y Bernbach, la publicidad nunca había vivido una transformación en el negocio tan profunda como en los últimos años. Hoy la propuesta de comunicación de la agencia para las marcas no había tenido tantas expectativas.

Actualmente los anunciantes valoran cambios en las metodologías publicitarias para tener resultados eficientes en sus marcas. Este modelo de planeación tiene valores adicionales en la realización de la investigación por el hallazgo en las cadenas de significación y enciclopedias, gran parte en el análisis semiótico y en su aplicación a la comunicación creativa. En este sentido, es interesante relacionar nuevas formas de hacer planeación estratégica y satisfacer las expectativas de los anunciantes ante las nuevas formas de trabajo y fuentes de información que indica la semiótica.

Según Pierce, las relaciones se pueden dividir en tres tricotomías las cuales funcionan perfectamente a las demandas de comunicación de las marcas: La primera (signo que es) tiene en cuenta el signo en sí mismo es decir ¿Qué quiere comunicar la marca?. La segunda (relación) tiene en cuenta la relación del signo con su objeto, es decir ¿Cómo se quiere comunicar el mensaje a las personas?. Y la tercera (como lo representa el interpretante) tiene en cuenta como si el interpretante lo representa como posibilidad, hecho o razón, es decir respuesta esperada por parte del interpretante de manera emocional. Esta propuesta que al analizarla y ejecutarla sobre el modelo de planeación da valor a las marcas.

Por esta razón, el modelo estratégico está construído en dos partes: en primer lugar el planning estratégico desde los estudios semióticos y en segundo lugar la eficacia de la comunicación. Así se trabaja en este modelo de planeación ya que mientras la investigación alimenta el análisis semiótico es más sólida, la eficacia en el mercado es más alta. Esta fórmula hace que el modelo sea exitoso.

Hoy junto al Profesor Vladimir Sánchez y un equipo interdisciplinario de profesores y estudiantes, se trabaja en el proyecto de investigación "Modelo semiótico de planeación estratégica publicitaria: Minería y análisis de datos con investigación de mercados y Big data como herramientas de planeación, control y prospectiva de resultados. Fase 1", financiado por la Universidad de Bogotá Jorge Tadeo Lozano. Este proyecto nace al determinar que una de las herramientas que más emplean los planners es el análisis de la información que proviene de Internet. El panorama de la planeación estratégica es cada vez más grande y profundo por la infinidad de datos que se encuentran en la red.

"La agencia de publicidad digital CP Proximity señala tres características principales del Big Data: manejar un gran volumen de información, procesar los datos a gran velocidad o en tiempo real, e integrar una gran variedad de fuentes de información que podrían generar conocimiento a partir de conexiones no evidentes (CP Proximity, 2012). Este es el estudio más destacado en el que el ámbito publicitario habla de Big Data. Por su parte, Harvard Business Review dedicó un especial sobre Big Data: The Management Revolution (McAfee, Brynjolfsson, 2012) y a su vez la consultora McKinsey publicó en mayo de 2011: Big data: the next frontier for innovation, competition and productivity (McKinsey Global Institute, 2011)”. (Sánchez-Blanco 2014) 
Aunque se ha empezado a investigar y a construir protocolos para determinar el alcance de Big Data en la planeación estratégica, es de suponer que se tendrá un gran papel en la comunicación publicitaria. Por esto se dan los pasos para que este modelo de planeación tenga una conexión interesante en la manera de involucrar este tipo de datos y así alimenten el proceso semiótico en el cual interactúan las personas analizando la manera en que se presentan y expresan, generando valiosos grounds para planear.

La proyección del futuro del modelo de planeación con los datos obtenidos ya sea por el análisis análogo o digital estará aportando a la relación entre la semiótica, la publicidad y la estrategia, dando paso a la academia que aporta a los procesos empresariales publicitarios, generando vínculos con la industria y modernizando los procesos de entrenamiento $\mathrm{y}$ de semilleros de los futuros publicistas.

\title{
Notas
}

1. Sánchez Riaño, V. (Septiembre 2011). LA CONSTRUCCIÓN DEL SENTIDO PUBLICITARIO: Modelo de análisis y evaluación desde la perspectiva semiótica de Charles Sanders Peirce. (Tesis de maestría). Universidad de Bogotá Jorge Tadeo Lozano, Colombia.

\section{Listas de Referencias bibliográficas}

Kavounas Taylor, A. (2013). Strategic Thinking. Pensamiento estratégico para creativos publicitarios. Londres. Laurence King Publishing. Ltd.

Moliné, M. (2000). La fuerza de la publicidad. Madrid: McGraw-Hill Latinoamericana de España.

Peirce, C.S. (2008). El pragmatismo. Madrid: Ediciones Encuentro, S.A.

Sánchez-Blanco, C. (2014). Relevancia del papel del planificador estratégico en la investigación publicitaria. Revista Mediterránea de Comunicación, vol. 5(1), 131-146. Disponible en http://mediterranea-comunicacion.org/. DOI 10.14198/MEDCOM2014.5.2.09.

Sánchez Riaño, V. (Septiembre 2011). LA CONSTUCCIÓN DEL SENTIDO PUBLICITARIO: Modelo de análisis y evaluación desde la perspectiva semiótica de Charles Sanders Peirce. (Tesis de maestría). Universidad de Bogotá Jorge

\begin{abstract}
The paper to present addresses some theoretical concepts of pragmatism and semiotics of Charles Sanders Peirce, in relation to the sign, the relationships established between signs and their projection to the Strategic Planning Advertising - Planning. Based on these theoretical elements, a semiotic model of strategic planning is proposed, resulting from the research project: "Semiotics, planning and advertising strategy, Pragmatism projections to contemporary communication" funded by the Jorge Tadeo Lozano University and executed in 2014-2015. The question that guides the presentation is: Which elements of Pragmatism and Peircean semiotics can be projected to a strategic planning model in advertising? For the development of the paper work three fundamental argu-
\end{abstract}


ments of pragmatism, known as phenomenological categories, which are related to the sensations, actions and thoughts that arise in the human mind. They are related between the feelings of the people, the actions they do and the thought of those actions and that in the advertising exercise are associated with the sensations that the consumers have against the products or brands, the purchase actions and the filiation processes with brands (strategic branding). This sequential approach allows proposing a strategic advertising planning methodology tied to measurable and efficient results. The model of Strategic Planning Advertising - Planning, was used with great success in 4 cases EFFIE College 2016 in Colombia. The result in this competition was: 2 finalists and one Gold in the case of the Bancolombia client.

Keywords: Semiotics - Peirce - advertising - strategic planning model

Resumo: Para apresentar trabalho discute alguns conceitos teóricos do pragmatismo e a semiótica de Charles Sanders Peirce, em relação ao sinal, as relações entre signos e sua projeção para a publicidade planejamento estratégico - Planning.

Palavras chave: Publicidade - planeamento - semiótica

[Las traducciones de los abstracts fueron supervisadas por el autor de cada artículo] 